\title{
A unusual case of 356 vesical calculii
}

\author{
A. Agarwal ${ }^{1}$, G. Sigdel ${ }^{1}$, S.R.K.C ${ }^{2}$, P.Shrestha ${ }^{2}$, W.K.Belokar ${ }^{3}$ \\ ${ }^{1}$ Mch Resident ${ }^{3}$ Professor and HOD, Department of Urology, CMS-TH Bharatpur, Nepal ${ }^{2}$ Resident, Department of Surgery, \\ CMS-TH Bharatpur, Nepal
}

\begin{abstract}
Multiple vesical calculi are rarely seen in urological practice. Males are affected more than the females. Vesical calculi are usually secondary to bladder outlet obstruction. These patients present with recurrent urinary tract infection, haematuria or with retention of urine. We report a 43 years male patient who presented with acute urinary retention. He had history of trauma over perineal region three years back following which he had recurrent urinary tract infection and thinning of stream. USG abdomen revealed normal upper urinary tract with echogenic debris in partially filled urinary bladder. Renal function test was with in normal limit. Per urethral catheterization failed and over antibiotic cover, patient was posted for cystourethroscopy followed by suprapubic cystostomy under spinal anaesthesia. Membranous urethral stricture found during urethroscopy could be managed by optical internal urethrotomy. On cystoscopy whole of bladder was filled with thick pus like material with multiple large urinary bladder calculi. Open cystolithotomy was done and we were surprised to see 356 stones of various size and shape after removal. Patient made uneventful recovery and discharged after 12 days of hospital stay.
\end{abstract}

Key words: Urinary stone, multiple vesical stone, urinary tract infection, urinary retention.

\section{Introduction}

Urinary bladder stone is commonly encountered in urology practice. The exact causes of the formation of bladder stones are not completely understood, but it usually occurs because of urinary tract infection (UTI), obstruction of the urinary tract, enlargement of the prostate gland in men, or the presence of foreign bodies in the urinary tract. Diet and the amount of fluid intake also appear to be important factors in the development of bladder stones. These are more common in men Correspondence: A. Agarwal E-mail: draaman@gmail.com and the majority of these are composed of triple phosphate. Multiple bladder stones have been reported several times in literature either as a sole entity or in association with foreign body ${ }^{1}$, cystocele ${ }^{2}$, migrated intrauterine contraseptic device ${ }^{1}$ etc. There are also reports of giant vesical calculus weighing more than $100 \mathrm{~g} .{ }^{3}$ Our case is unique because such a large number of stones(356) removed from urinary bladder at a time has never been mentioned in any literature. Available treatment options for vesical calculi include open surgical removal, 
A. Agarwal, A unusual case of 356 vesical calculii

extracorporeal fragmentation and endoscopic crushing followed by extraction of pieces. Recently, endosurgical mechanical cystolithotripsy followed by percutaneous extraction has been evolved for small or moderatesized calculi. We managed our case successfully by open cystolithotomy.

\section{Case report}

A 43-year-old male presented in college of medical sciences, Bharatpur, Nepal with inability to pass urine from last 6 hours. On arrival he was afebrile and his vitals were stable. History revealed that he suffered from perineal trauma three years back and from last one year he was having recurrent episode of UTI and progressive thinning of urinary stream. On abdominal examination mild suprapubic fullness with out any tenderness was present and per rectal examination was unequivocal. Renal function test was in normal limit and USG abdomen revealed normal upper urinary tract with echogenic debris in partially filled urinary bladder. Suprapubic aspiration for urine examination and culture sensitivity, suggested frank pyuria. Urine examination showed plenty of WBCs. Attempt to pass per urethral catheter failed. Provisional diagnosis of urethral stricture was made and patient was planned to go for urethroscopy with optical internal urethrotomy (OIU), if possible, other wise suprapubic cystostomy, on the same day. Over antibiotic cover and under spinal anesthesia urethroscopy revealed superficial, passable, membranous urethral stricture easily managed by OIU. On further negotiation of 23 charr cystoscope multiple pale colored concretions were found in prostatic urethra which were pushed back inside bladder. On cystoscopy whole of the bladder was found to be filled with thick pus like material and there was no visibility et al.
Bladder wash was done multiple times till returning fluid became clear. Multiple small stones could be seen coming out along with the returning fluid through ellik evacuator. Repeat cystoscopy showed multiple stones in bladder of different size and shape, however no diverticulum was seen. No attempt was made to remove these stones endosmotically because stones were infected, large sized and multiple. Open cystolithotomy (fig1) was done and to our surprise 356 stones of various size and shape (fig2) were removed along with some blood clots. Bladder was close with suprabupic and per urethral catheter in situ.

Removed stones on gross examination were dirty pale in color, 356 in number(excluding stones which were less than 4mm), of different shapes as shone in figure 2 , and of size ranging from $4 \mathrm{~mm}$ to $28 \mathrm{~mm}$ in largest dimension. Biochemical examination revealed triple phosphate composition of stones.

Urine culture grew E-coli and patient made uneventful recovery and was discharged after 12 days of hospital stay. Above case in unique because USG failed to demonstrate any stone in urinary bladder and the diagnosis could be made only after cystoscopy, secondly such a large number of stones removal, at a time, is not mentioned in any literature and thirdly it gave us the message that in all the cases of urinary retention x-ray KUB should be done, which we failed to do.

\section{Discussion}

Urinary bladder stone accounts for only $5 \%$ of all urinary calculi. ${ }^{2}$ Even more rare are multiple bladder stones and giant vesical calculi weighing more than 
$100 \mathrm{~g} .{ }^{4}$ Though in developing countries children with vesical stones often present with a large stone burden. ${ }^{6}$ Females are generally less commonly affected than males as ninety-five percent of all bladder stones occur in men. ${ }^{2,6}$ Bladder calculi most often occur in conditions of chronic urinary retention, bladder outlet obstruction or urinary tract infection. ${ }^{2,6}$ These conditions are commonly precipitated by development of a bladder diverticulum ${ }^{5,6}$ benign prostatic hypertrophy in males, genital prolapse in females ${ }^{2}$ or urethral stricture. Prolonged catheterisation, neurogenic bladder, foreign body and trauma are other rarer causes. ${ }^{6}$ There are case reports of formation of bladder stones around a foreign body, sutures, catheters or other objects introduced into the bladder which act as a nidus for stone formation. ${ }^{2}$ Pomerantz et al. have reported a rare case of formation of urinary bladder calculus around an arterial graft, which was incorporated in the bladder. ${ }^{6}$ It is thought that vesical calculus develops from the nidus of the infected material or from a single ureteric calculus with progressive layer-wise deposition of calcified matrix. Thus, each of the earlier stated factors may mutually contribute to the formation of a calculus. ${ }^{7}$ Also, studies have indicated that infection may not be the inciting factor in stone formation, but may play a major role in further stone crystallization. ${ }^{2}$

Most of the vesical calculi are composed of triple phosphate, calcium carbonate, and calcium oxalate. Presentation of patients with vesical calculus is commonly with recurrent urinary tract infection, hematuria, inability to pass urine and azotaemia. ${ }^{8}$ Complaints of suprapubic discomfort or pain may or may not be there. Patients in urinary retention caused by a vesical calculus usually pass urine in supine position. Chronic obstruction to urine flow due to a vesical calculus further leads to infection, and, rarely, bladder perforation ${ }^{9,10}$ hydronephrosis, and acute renal failure. ${ }^{5,8}$

The majority of bladder calculi are radiopaque and detected by plain radiograph. Other investigations which can show bladder calculi are ultrasound, CTscan, magnetic resonance imaging and intravenous urogram but contrast-enhanced $\mathrm{CT}$ is the investigation of choice as it has remarkable sensitivity in detecting urinary tract stones, including uric acid stones. It can reveal the concentric nature of stones.

Various surgical modalities are available for vesical calculi which include open surgical removal, extracorporeal fragmentation and endoscopic crushing followed by extraction of pieces. Electrohydraulic shockwave lithotripsy (EHSWL) is preferably to be avoided in hard, large vesical calculi and if the stone is in a diverticulum or stuck to the mucosa. ${ }^{11}$

Recently, endosurgical mechanical cystolithotripsy followed by percutaneous extraction has been evolved for small or moderate-sized calculi. It has an added advantage as it can be combined with a corrective procedure for bladder outlet obstruction. ${ }^{12}$ Isen et al reported their experience in the management of bladder stones by pneumatic lithotripsy using a ureteroscope in boys 3 to 14 years old with bladder stones ${ }^{13}$ Percutaneous cystolithotomy is also considered safe alternative, with low morbidity and a low complication rate for large burden vesical stones. ${ }^{14}$ 
A. Agarwal, A unusual case of 356 vesical calculii

Figure 1: Cystostomy showing multiple vesical calculii

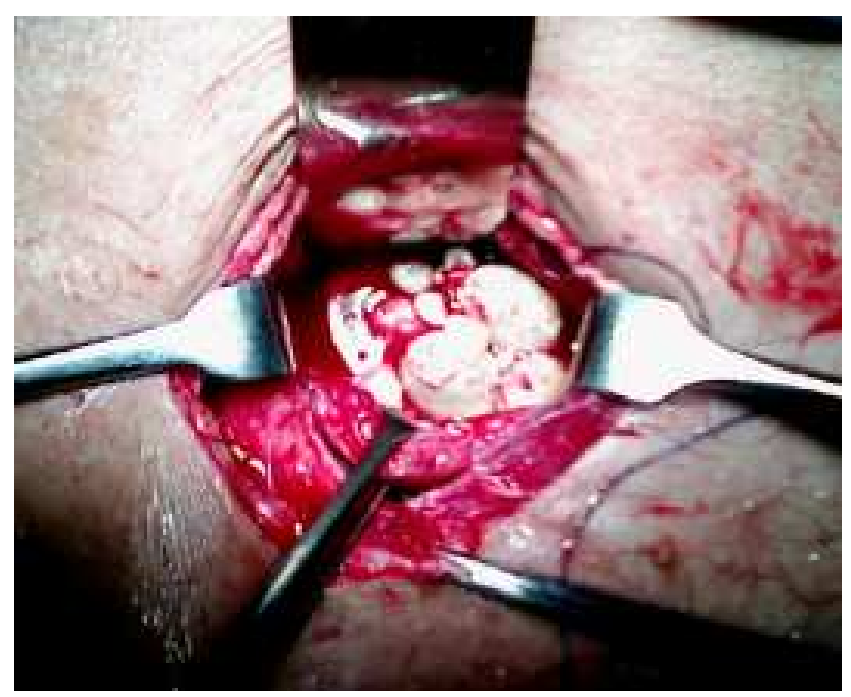

Figure 2: dirty pale, different size and shape, multiple vesical calculi specimen

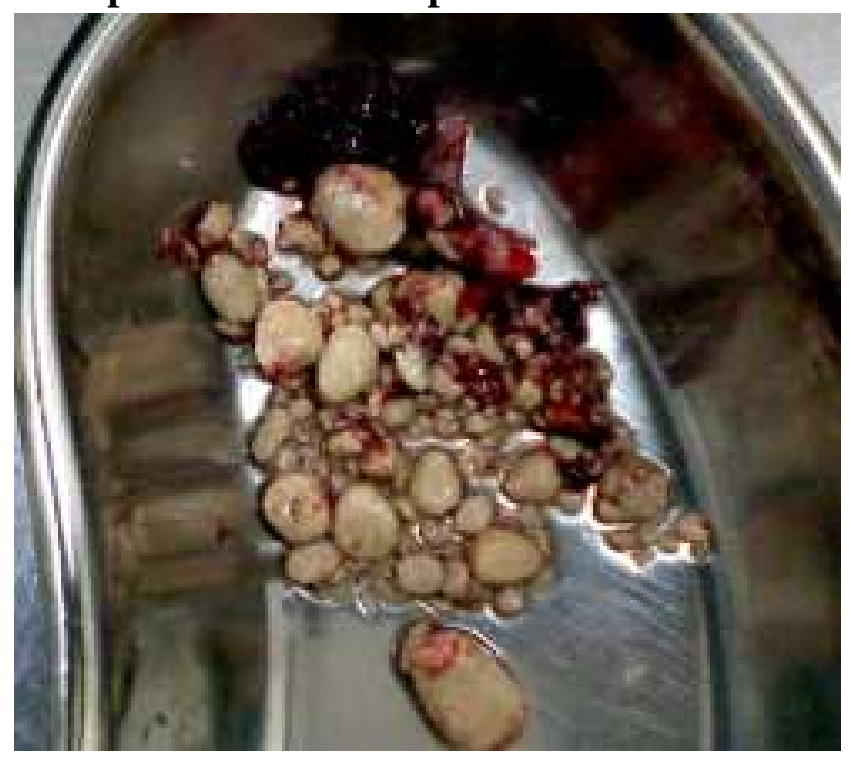

\section{Conclusion}

Though different modalities of treatment are available in literature for removing vesical calculus, but enthusiasm of using minimally invasive modality in treatment of multiple and infected vesical calculi may produce unwanted complications. Open cystolithotomy is a quick and safe procedure to deal with these type of stones and can always be preferentially considered over any other minimally invasive procedure.

\section{References}

1. A.Ozgür, A.Sismanoglu, C.Yazici, et al. Intravesical stone formation on intrauterine contraceptive device. Int Urol Nephrol 2004;36 (3):345-8.

2. B.F.Schwartz, M.Z.Stoller. The vesical calculus. Urol Clin North Am 2000; 27: 333-46.

3. G.Shilpi, S.Onkar, S.Sumit, et al. Giant Vesical Calculus. The Internet Journal of Surgery 2008; 17: 1.

4. R.M.Becher, B.M.Tolia, H.R.Newman. Giant vesical calculus. JAMA 1976; 239: 2272-3.

5. S.K.Mishra, A.Ganpule, T.Manohar,et al. Surgical management of pediatric urolithiasis. Indian $J$ Urol 2007; 23: 428

6. P.A.Pomerantz. Giant vesical calculus formed around arterial graft incorporated into bladder. Urology 1989; 33: $57-8$.

7. G.E.Leach, T.J.Fitzpatrick. Giant vesical calculi in the female. Urology 1981; 17: 274-5.

8. P.N.Maheshwari, A.T.Oswal, M.Bansal. Percutaneous cystolithotomy for vesical calculi: a better approach. Tech-Urol 1999; 5: 40-2.

9. N.Kaur, A.Attam, A.Gupta,et al. Spontaneous bladder rupture caused by a giant vesical calculus. Int Urol Nephrol 2006; 38: 487-9.

10. A.Basu, I.Mojahid, E.P.Williamson. Spontaneous bladder rupture resulting from giant vesical calculus. Br J Urol 1994; 74: 385-6.

11. Z.Zhaowu, Xiwen, Z.Fenling. Experience with electrohydraulic shockwave lithotripsy in the treatment of vesical calculi. BJU 1988; 61: 498-9.

12. R.Asci, Z.Aybek, S.Sarikaya,et al. The management of vesical calculi with optical mechanical cystolithotripsy and transurethral prostatectomy: is it safe and effective? BJU Inter 1999; 84: 332-6.

13. K.Isen, S.Em, V.Kilic, et al. Management of bladder stones with pneumatic lithotripsy using a ureteroscope in children. J Endourol 2008; 22: 1037.

14. F.Demirel, M.Cakan, F.Yalcinkaya,et al. Percutaneous suprapubic cystolithotripsy approach: for whom? Why? J Endourol 2006; 20: 429. 